\title{
Betaine alleviates hepatic lipid accumulation via enhancing hepatic lipid export and fatty acid oxidation in rats fed with a high-fat diet
}

\author{
Li Xu, Danping Huang, Qiaolin Hu, Jing Wu, Yizhen Wang and Jie Feng* \\ Key Laboratory of Molecular Animal Nutrition, Ministry of Education, College of Animal Sciences, Zhejiang University, \\ Hangzhou 310029, People's Republic of China \\ (Submitted 15 October 2014 - Final revision received 9 February 2015 - Accepted 11 March 2015 - First published online 29 April 2015)
}

\section{Abstract}

To assess the effects of betaine on hepatic lipid accumulation and investigate the underlying mechanism, thirty-two male Sprague-Dawley rats weighing 100 (SD 2.50) g were divided into four groups, and started on one of four treatments: basal diet, basal diet with betaine administration, high-fat diet and high-fat diet with betaine administration. The results showed that no significant difference of body weight was found among experimental groups. Compared with high-fat diet-fed rats, a betaine supplementation decreased $(P<0 \cdot 05)$ hepatic TAG accumulation induced by high-fat diet, which was also supported by hepatic histology results. Additionally, hepatic betaine-homocysteine methyltransferase activity as well as its mRNA abundance and lecithin level were found increased $(P<0.05)$ by betaine supplementation in both basal diet-fed rats and high-fat diet-fed rats. Betaine administration in high-fat diet-fed rats exhibited a higher $(P<0.05)$ activity of hepatic carnitine palmitoyltransferase 1 (CPT1) compared with high-fat diet-fed rats. High-fat diet inhibited $(P<0 \cdot 05)$ the gene expression of hepatic $P P A R \alpha$ and $C P T 1$. However, betaine administration in high-fat diet-fed rats elevated $(P<0.05)$ the gene expression of $P P A R \alpha$ and $C P T 1$. Moreover, concentration, gene and protein expressions of hepatic fibroblast growth factor 21 (FGF21) were increased $(P<0.05)$ in response to betaine administration in high-fat diet group; meanwhile the gene expression of hepatic AMP-activated protein kinase was increased $(P<0 \cdot 05)$ as well. The results suggest that betaine administration enhanced hepatic lipid export and fatty acid oxidation in high-fat diet-fed rats, thus effectively alleviating fat accumulation in the liver.

Key words: Betaine: Liver: Lipid accumulation: High-fat diet: Rats

Betaine ( $N, N, N$-trimethylglycine) that serves as an effective methyl donor and an osmolyte is commonly used in animals and human beings for the purpose of sparing dietary methionine, and helping to treat homocysteinaemia separately ${ }^{(1,2)}$. In vivo, betaine plays a key role in sulphur-amino acid metabolism, and is metabolised to dimethylglycine and sarcosine ${ }^{(3)}$. Numerous studies indicate that betaine has important nutritional and physiological functions ${ }^{(4-8)}$, such as growth promotion, anti-stress effect, reproductive performance improvement, antioxidant preventive effect as well as osmotic protection. Furthermore, studies on swine and poultry have suggested that betaine supplementation can decrease overall fat deposition, and improve carcass characteristics by stimulating lipolysis ${ }^{(9-11)}$. Clinical studies have shown that betaine might serve as a safe and promising therapeutic agent for nonalcoholic fatty liver disease and nonalcoholic steatohepatitis in human beings ${ }^{(12,13)}$. Lipid accumulation results from an imbalance among the synthesis, oxidation and transportation of fatty acids ${ }^{(14-17)}$, and plays a key role in disease initiation, which can be seen in many chronic liver diseases. Therefore, the beneficial effects of betaine in preventing hepatic fat accumulation have received much attention in recent years. In rats and human subjects, high-fat diet, ethanol as well as high sucrose induced hepatic lipid accumulation and liver injury, and these changes could be reversed by the administration of betaine ${ }^{(18-20)}$. Previous studies indicated that betaine triggers hepatoprotective effect mostly through alleviating impairment of sulphur-amino acid metabolism and oxidative stress ${ }^{(21-23)}$.

However, the effects of betaine on hepatic lipid accumulation and molecular mechanisms behind these alterations were not fully clarified. Therefore, the present study focused on the effects of betaine on hepatic lipid accumulation, and investigated the regulation interrelationship between betaine and related key factors involved in protective effects against abnormal lipid accumulation in Sprague-Dawley rats.

\section{Materials and methods}

\section{Animal experimental procedure}

The present study was approved by the Institutional Animal Care and Use Committee of Zhejiang University

Abbreviation: CPT1, carnitine palmitoyltransferase 1.

*Corresponding author: Professor J. Feng, email fengj@zju.edu.cn 
(Hangzhou, China). The experiment was conducted in the Laboratory Animal Center of Zhejiang University, and all necessary precautions were taken to mitigate pain. SpragueDawley rats used for the investigation were purchased from Medical Animal Laboratory Center of Zhejiang Chinese Medical University (Hangzhou, China). Thirty-two 3-week-old male Sprague-Dawley rats (initial weight: 100 (SD 2.50) g) were randomly divided into four different groups: basal diet (T1), basal diet with betaine administration (T2), high-fat diet (T3) and high-fat diet with betaine administration (T4). $\mathrm{T} 1$ and T2 were given basal diet (Table 1), while T3 and T4 were given high-fat diet (Table 1). Meanwhile, T2 and T4 were administrated intragastrically with $1 \mathrm{ml}$ of betaine $(98 \%$ of purity; Sigma-Aldrich) at a concentration of $400 \mathrm{mg} / \mathrm{kg}$ at 17.00 hours per $\mathrm{d}$, while T1 and T3 groups were given $1 \mathrm{ml}$ of $0.9 \%$ saline by oral administration. The energy compositions of basal diet were $13.8 \%$ derived from fat, $25 \cdot 7 \%$ from protein and $60.5 \%$ from carbohydrates (Table 1). In high-fat diet, $40.0 \%$ of its energy derived from fat, $20.0 \%$ from protein and $40.0 \%$ from carbohydrates (Table 1). The diets were formulated according to AIN-93G (American Institute of Nutrition recommendation for laboratory rodents) ${ }^{(24)}$ The basal and high-fat diets were prepared specifically for our study by Slac Experimental Animal LLC (Shanghai, China). A schedule of $12 \mathrm{~h}$ light and $12 \mathrm{~h}$ darkness was used for the Sprague-Dawley rats. All rats were individually housed in a stainless steel cage and had free access to chow diet and water throughout the entire feeding period. Body weights of rats were recorded weekly. The experiments lasted for $28 \mathrm{~d}$.

Table 1. Nutrition formulation of diet*

\begin{tabular}{|c|c|c|}
\hline & Basal diet & High-fat diet \\
\hline \multicolumn{3}{|l|}{ Ingredient ( $\mathrm{g} / \mathrm{kg})$} \\
\hline Maize starch & 504.48 & 293.24 \\
\hline Casein & $230 \cdot 00$ & $227 \cdot 58$ \\
\hline Sucrose & $100 \cdot 00$ & 194.60 \\
\hline Soyabean oil & $60 \cdot 00$ & $32 \cdot 76$ \\
\hline Lard oil & 0 & 169.00 \\
\hline Fibre & $50 \cdot 00$ & $27 \cdot 30$ \\
\hline Mineral mix $\dagger$ & 35.00 & 35.00 \\
\hline Vitamin mixt & $10 \cdot 00$ & $10 \cdot 00$ \\
\hline L-Cys & 3.00 & 3.00 \\
\hline Choline bitartrate & 2.50 & 2.50 \\
\hline Antioxidant & 0.02 & 0.02 \\
\hline \multicolumn{3}{|l|}{ Nutrient level (g/kg) } \\
\hline Carbohydrate & 520.00 & $446 \cdot 00$ \\
\hline Crude protein & 221.00 & 223.00 \\
\hline Crude lipid & $52 \cdot 80$ & 198.00 \\
\hline \multicolumn{3}{|c|}{ Fatty acid composition (\%) } \\
\hline C14:0 & $0 \cdot 10$ & 0.90 \\
\hline C16:0 & $9 \cdot 90$ & $20 \cdot 20$ \\
\hline $\mathrm{C} 16: 1$ & 0.20 & 3.80 \\
\hline C18:0 & 3.70 & 9.90 \\
\hline C18: $1 n-9$ & 21.40 & $36 \cdot 80$ \\
\hline C18:2n-6 & $54 \cdot 10$ & 24.60 \\
\hline C18:3n-3 & 8.80 & $3 \cdot 10$ \\
\hline $\mathrm{C} 20: 1 n-9$ & $1 \cdot 30$ & 0.30 \\
\hline
\end{tabular}

* The data of nutrient level and fatty acid composition were analysed values. † Vitamin mix and mineral mix are in accordance with the American Institute of Nutrition-93 guidelines.

\section{Sampling}

The rats were anesthetised with halothane after the $28 \mathrm{~d}$ intragastric administration trial. Orbital blood was collected into coagulation promoting tubes and centrifuged at $3000 \mathrm{~g}$ for $10 \mathrm{~min}$ at $4^{\circ} \mathrm{C}$. Then the serum was extracted and stored at $-80^{\circ} \mathrm{C}$. The rats were then killed by cervical dislocation, and liver samples were collected. About $2 \mathrm{~cm}$-wide of fresh liver chop was collected in a CryoTube (Corning) and frozen in liquid $\mathrm{N}_{2}$ for Oil-Red-O histological assessment, another specimen of liver fixed in $10 \%$ formaldehyde for haematoxylin-eosin stain. The remainders were snap-frozen in liquid $\mathrm{N}_{2}$, and stored at $-80^{\circ} \mathrm{C}$ for subsequent analysis of lipid metabolites, mRNA and protein expression.

\section{Analysis of lipid metabolites in serum}

Serum TAG, total cholesterol, NEFA and glucose were measured by using the commercial analysis kits (JianCheng Institute of Biotechnology). The serum levels of lecithin and VLDL were determined by using ELISA kits (A\&D Company Limited) according to manufacturer's instructions.

\section{Hepatic histology and hepatic lipid metabolites analysis}

After being fixed in $10 \%$ formaldehyde for $24 \mathrm{~h}$ and dehydrated, specimens of liver were embedded in paraffin, sliced into sections of $4 \mu \mathrm{m}$ thickness and stained with haematoxylin-eosin. For Oil-Red-O assessment, $10 \mu \mathrm{m}$ frozen sections of liver tissue were immersed in isopropanol for $1 \mathrm{~min}$ and stained with Oil-Red-O for $10 \mathrm{~min}$. After being washed three times with distilled water, the sections were counterstained in haematoxylin, and washed again in distilled water before microscopic analysis. The positive result of Oil-Red-O staining was bright red. Software of Image pro-plus 6.0 (Media Cybernetics) was used to estimate the positive ratio that described the percentage of the red area in each image. The levels of TAG, total cholesterol, NEFA, lecithin, VLDL and fibroblast growth factor 21 (FGF21) in liver were also measured by using the commercial analysis kits as mentioned above. Hepatic carnitine, carnitine palmitoyltransferase 1 (CPT1) and betaine-homocysteine methyltransferase (BHMT) levels were determined by using ELISA kits (A\&D Company Limited) according to manufacturer's instructions.

\section{Analysis of gene expression}

Total RNA of liver and cells were extracted by using Trizol (Invitrogen) according to the protocol provided by the manufacturer. Isolated RNA was quantified by using the NanoDrop ND-2000 spectrophotometer (Thermo Fisher Scientific, USA), and its integrity was confirmed by agarose gel electrophoresis. The complementary DNA (cDNA) synthesis was performed in a $20 \mu \mathrm{l}$ reaction volume containing $2 \mu \mathrm{g}$ total RNA, $1 \mu \mathrm{l}$ random hexamer, $4 \mu \mathrm{l}$ of five reaction buffer, $2 \mu \mathrm{l}$ of $10 \mathrm{mmol} / \mathrm{l}$ deoxyribonucleotide triphosphate (dNTP) and $10 \mu \mathrm{l}$ of 200 units $/ \mu \mathrm{l}$ Moloney murine leukaemia virus (M-MLV). And the abundance of target gene was measured by quantitative real-time PCR analysis, using the ABI StepOne Plus ${ }^{\text {TM }}$ RT-PCR system with 
Table 2. Primer-pairs of target genes used for real-time PCR

\begin{tabular}{|c|c|c|c|}
\hline Gene & $\begin{array}{l}\text { Forward primer (from } 5^{\prime} \text { to } 3^{\prime} \text { ) } \\
\text { Reverse primer (from } 5^{\prime} \text { to } 3^{\prime} \text { ) }\end{array}$ & PCR product size $(\mathrm{bp})$ & GenBank accession number \\
\hline$\beta$-Actin & $\begin{array}{l}\text { GGA AAT CGT GCG TGA CAT TA } \\
\text { AGG AAG GAA GGC TGG AAG GAG }\end{array}$ & 183 & NM_031144 \\
\hline BHMT & $\begin{array}{l}\text { GGGCAGAAGGTCAATGAAGCT } \\
\text { ACCAATGCATCCCCTTCGT }\end{array}$ & 108 & NM_030850 \\
\hline$P P A R \alpha$ & $\begin{array}{l}\text { TGCGGACTACCAGTACTTAG } \\
\text { CGACACTCGATGTTCAGTGC }\end{array}$ & 167 & M88592 \\
\hline FGF21 & $\begin{array}{l}\text { CGACAGAGGTATCTCTACACAGATGACG } \\
\text { GATCCATAGAGAGTTCCATCTGGTTGTT }\end{array}$ & 206 & NM_130752 \\
\hline$A M P K$ & $\begin{array}{l}\text { TGTGACAAGCACATTTTCCAA } \\
\text { CCGATCTCTGTGGAGTAGCAG }\end{array}$ & 156 & NM_019142.2 \\
\hline CPT1 & $\begin{array}{l}\text { GCTCGCACATTACAAGGACAT } \\
\text { TGGACACCACATAGAGGCAG }\end{array}$ & 250 & AF020776 \\
\hline
\end{tabular}
carnitine palmitoyltransferase 1.

the SYBR $^{\circledR}$ Premix Ex Taq $^{\text {TM }}$ (Tli RNaseH Plus) RT-PCR kit (TaKaRa), according to the manufacturer's instructions. The primer pairs of specific genes were commercially synthesised by TaKaRa, shown in Table 2 . Each sample was analysed in duplicate and the expression of the target genes were standardised by the endogenous housekeeping gene ( $\beta$-actin). The reaction protocol comprised one cycle of $95^{\circ} \mathrm{C}$ for $2 \mathrm{~min}$, thirty-five cycles of $94^{\circ} \mathrm{C}$ for $50 \mathrm{~s}, 60^{\circ} \mathrm{C}$ for $30 \mathrm{~s}$ and $72^{\circ} \mathrm{C}$ for $50 \mathrm{~s}$. The gene expression was calculated by using the comparative $\left(2^{-\Delta \Delta C_{\mathrm{T}}}\right)$ method $^{(25,26)}$.

\section{Western blot analysis}

Protein of liver samples was extracted and quantified by using a Bradford protein assay kit (Bio-Rad). Briefly, liver samples were homogenised at $4^{\circ} \mathrm{C}(1: 10, \mathrm{w} / \mathrm{v})$ in $50 \mathrm{~mm}$-Tris- $\mathrm{HCl}$ buffer ( $\mathrm{pH}=8 \cdot 0$ ), containing $1 \mathrm{mmol} / \mathrm{l}$ phenylmethylsulfonyl fluoride, $1 \mathrm{mg} / \mathrm{ml}$ protease inhibitor cocktail, $0 \cdot 1 \% \beta$-mercaptoethanol, and centrifuged at $12000 \mathrm{~g}$ at $4^{\circ} \mathrm{C}$ for $5 \mathrm{~min}$. After being heated to $95^{\circ} \mathrm{C}$ for $5 \mathrm{~min}$, proteins were separated on SDS-PAGE gels, and then transferred onto immobilon-P polyvinylidene difluoride membranes (Millipore). After blocking with 5\% non-fat milk/Tween overnight, the membranes were immunoblotted with the antibodies specifiedanti-FGF-21 antibody (Epitomics), and a horseradish peroxidase-conjugated secondary antibody (KPL) was used in the detection of specific proteins. For examining the equal loading, $\beta$-actin antibody (Santa Cruz Biotechnology) was used as control. Finally, enhanced chemiluminescence reagent (Amersham) was used to visualise the protein bands. Band intensities were determined by using AlphaEase FC analysis software (version 4; Alpha Innotech).

\section{Statistical analysis}

Data analyses were performed with the SPSS 20.0 statistical software package (IBM). Statistical analysis was performed using one-way ANOVA, and was analysed further by Duncan's multiple range test for differences among groups. Results were presented as means and standard deviations. In all analyses, the level of significant difference was set at $P<0.05$.

\section{Results}

\section{Assessment of body weight}

Changes of body weight were shown in Table 3. The body weights of rats in high-fat group were numerically lower than those in other groups since the 14th day, but no significant difference $(P>0.05)$ was found in body weight among experimental groups at the end of the feeding period.

\section{Effects of betaine on serum lipid metabolites}

As shown in Table 4, compared with rats fed with the basal diet (T1), 4 weeks of high-fat diet (T3) feeding significantly elevated

Table 3. Changes of body weight during 4 weeks (g) (Mean values and standard deviations, $n 7$ )

\begin{tabular}{|c|c|c|c|c|c|c|c|c|}
\hline & \multicolumn{2}{|c|}{$\mathrm{T} 1$} & \multicolumn{2}{|c|}{ T2 } & \multicolumn{2}{|c|}{ T3 } & \multicolumn{2}{|c|}{ T4 } \\
\hline & Mean & SD & Mean & SD & Mean & SD & Mean & SD \\
\hline $\mathrm{Od}$ & $100 \cdot 02$ & 0.15 & $100 \cdot 15$ & 0.28 & 99.91 & 0.13 & 99.95 & 0.36 \\
\hline $7 d$ & $150 \cdot 00$ & 2.58 & 148.75 & 3.14 & $152 \cdot 12$ & 3.46 & $149 \cdot 12$ & 3.03 \\
\hline $14 \mathrm{~d}$ & $202 \cdot 00$ & 5.42 & 205.5 & 6.75 & $207 \cdot 75$ & 7.45 & $206 \cdot 17$ & $7 \cdot 60$ \\
\hline $21 \mathrm{~d}$ & $228 \cdot 74$ & 16.83 & $244 \cdot 13$ & 17.55 & $227 \cdot 61$ & $26 \cdot 12$ & $253 \cdot 83$ & 31.47 \\
\hline $28 d$ & 290.58 & $9 \cdot 86$ & 296.03 & $14 \cdot 26$ & $287 \cdot 26$ & $15 \cdot 80$ & $305 \cdot 75$ & $25 \cdot 67$ \\
\hline
\end{tabular}

T1, basal diet; T2, basal diet with betaine administration; T3, high-fat diet; T4, high-fat diet with betaine administration. 
Table 4. Effects of betaine on serum lipid metabolism

(Mean values and standard deviations, $n 7$ )

\begin{tabular}{|c|c|c|c|c|c|c|c|c|}
\hline & \multicolumn{2}{|c|}{$\mathrm{T} 1$} & \multicolumn{2}{|c|}{ T2 } & \multicolumn{2}{|c|}{ T3 } & \multicolumn{2}{|c|}{ T4 } \\
\hline & Mean & SD & Mean & SD & Mean & SD & Mean & SD \\
\hline TAG (mmol/l) & $0.90^{c}$ & 0.11 & $0.92^{c}$ & $0 \cdot 10$ & $1 \cdot 38^{b}$ & 0.20 & $1.60^{\mathrm{a}}$ & 0.16 \\
\hline NEFA $(\mu \mathrm{mol} / \mathrm{l})$ & 234.90 & 71.98 & 246.73 & 83.36 & 288.93 & $77 \cdot 71$ & 31.4 .96 & 75.78 \\
\hline $\mathrm{TC}(\mathrm{mmol} / \mathrm{l})$ & 1.94 & 0.16 & 1.98 & 0.22 & 2.06 & 0.15 & 2.15 & 0.21 \\
\hline Glucose $(\mathrm{mmol} / \mathrm{l})$ & $3.75^{\mathrm{d}}$ & 0.46 & $4 \cdot 56^{c}$ & 0.51 & $5 \cdot 15^{\mathrm{b}}$ & 0.32 & $5 \cdot 86^{\mathrm{a}}$ & 0.39 \\
\hline Lecithin $(\mathrm{ng} / \mathrm{ml})$ & $9.57^{d}$ & 0.23 & $13.69^{c}$ & 0.66 & $15 \cdot 83^{\mathrm{b}}$ & 0.86 & $17 \cdot 36^{\mathrm{a}}$ & 0.33 \\
\hline $\operatorname{VLDL}(\mu \mathrm{mol} / \mathrm{l})$ & $51 \cdot 88^{b}$ & 4.26 & $54 \cdot 13^{\mathrm{b}, \mathrm{c}}$ & 3.67 & $57.89^{\mathrm{a}, \mathrm{c}}$ & $3 \cdot 24$ & $59 \cdot 20^{\mathrm{a}}$ & 5.58 \\
\hline
\end{tabular}

$\mathrm{T} 1$, basal diet; T2, basal diet with betaine administration; T3, high-fat diet; T4, high-fat diet with betaine administration; TC, total cholesterol.

a,b,c,d Mean values with unlike superscript letters were significantly different $(P<0.05)$.

$(P<0 \cdot 05)$ the concentration of serum TAG, glucose, lecithin and VLDL. Compared with the rats fed with the high-fat diet (T3), the levels of serum TAG, glucose and lecithin were increased $(P<0.05)$ further in the high-fat diet group with betaine administration (T4). Furthermore, betaine administration in basal diet-fed rats (T2) significantly increased $(P<0.05)$ the levels of glucose and lecithin compared with the rats fed with the basal diet (T1). Moreover, betaine administration groups (T2 and T4) had numerically higher levels of VLDL. No significant differences were found in total cholesterol and NEFA levels among experimental groups.

\section{Oral administration of betaine effectively alleviated the excessive accumulation of fat in the liver}

The high-fat diet feeding for 4 weeks caused hepatic lipid accumulation in rats (Figs. 1 and 2), which was illustrated by severe vacuolar degeneration in haematoxylin-eosin-stained sections, and intensive bright red staining in Oil-Red-O-stained sections respectively (Figs. 1(C) and 2(C)). Oral administration of betaine in rats fed with the high-fat diet (T4) reduced $(P<0.05)$ hepatic accumulation of lipids (Figs. 1(D) and 2(D)). The differences in vesicular fat accumulations between histological sections staining with Oil-Red-O were confirmed by image analysis. Consistent with the results of hepatic histology, high-fat diet administration (T3) increased $(P<0.05)$ hepatic levels of TAG and NEFA (Table 5). Oral administration of betaine did not affect TAG concentration in rats fed with the basal diet, but a significant reduction $(P<0.05)$ in hepatic TAG content was observed in betaine-administered rats fed with the high-fat diet (T4) (Table 5).

Betaine elevated the activity and the mRNA expression of betaine-homocysteine methyltransferase in the liver

Compared with groups without betaine administration (T1 and T3), betaine supplementation groups (T2 and T4) exhibited a significant increase $(P<0.05)$ in the activity of BHMT in the liver (Fig. 3(A)). High-fat diet also elevated $(P<0 \cdot 05)$ the activity of BHMT when compared with rats fed with basal diet. Furthermore, the gene expression of $B H M T$ in high-fat diet-fed rats was elevated $(P<0.05)$ by betaine administration whereas the changes of BHMT gene expression in basal diet rats were not observed (Fig. 3(B)).

Betaine increased PPAR $\alpha$ gene expression and reversed the inhibition of carnitine palmitoyltransferase 1 gene expression induced by high-fat diet in the liver

Effects of betaine administration on the activity of CPT1 and the gene expressions of PPAR $\alpha$ and CPT1 were shown in Fig. 4. Betaine administration in rats fed with a high-fat diet exhibited a higher activity of CPT1 $(P<0.05)$ compared with that of high-fat diet-fed rats (Fig. 4(A)). Compared with rats fed with basal diet, high-fat diet supplementation significantly suppressed the gene expression of PPAR $\alpha$ and CPT1 in the liver $(P<0.05)$. In high-fat group with betaine administration, the inhibition induced by high-fat diet was reversed, oral administration of betaine significantly increasing $(P<0.05)$ the gene expression of $P P A R \alpha$ and CPT1 in the liver. Moreover, betaine administration rats fed with basal diet also showed elevated $(P<0.05) P P A R \alpha$ mRNA abundance, but it had no influence on the mRNA abundance of CPT1 (Fig. 4(B) and (C)).
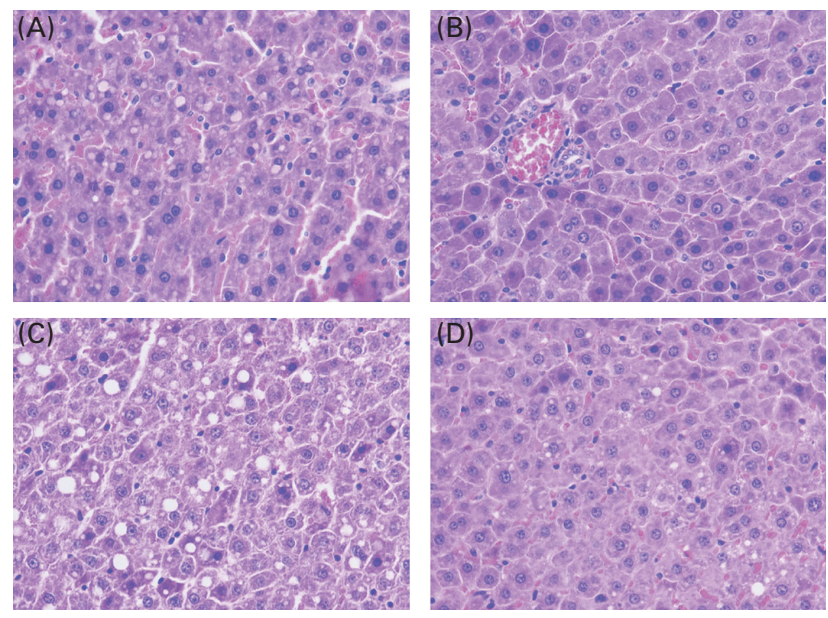

Fig. 1. Haematoxylin-eosin-stained liver sections of Sprague-Dawley rats from: (A) basal diet; (B) basal diet with betaine administration; (C) high-fat diet; (D) high-fat diet with betaine administration. A colour version of this figure can be found online at http://www.journals.cambridge.org/bjn 

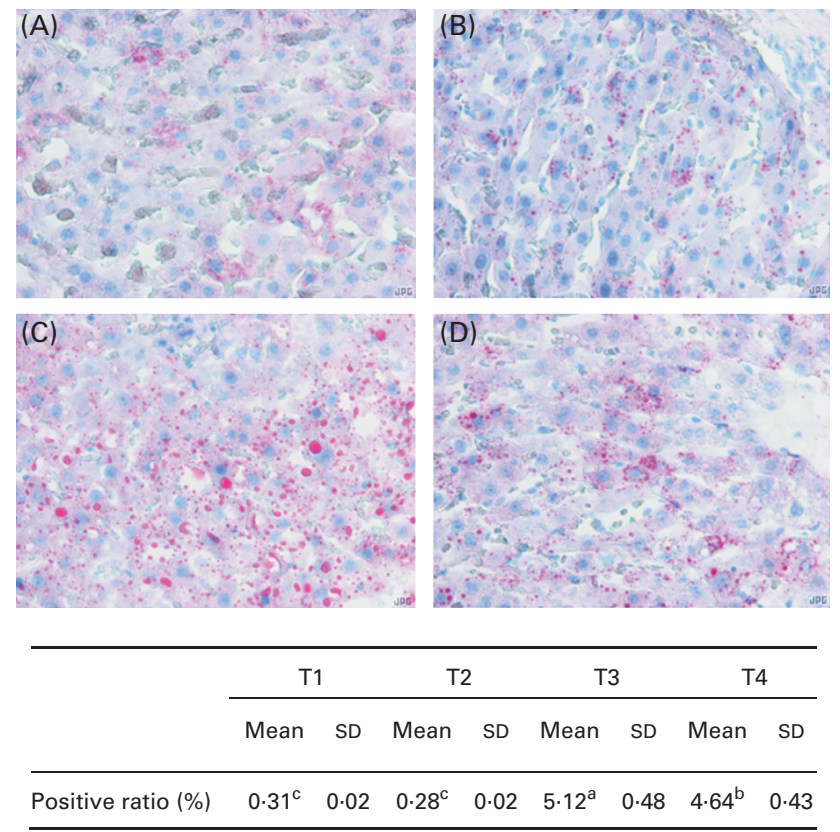

Fig. 2. Oil-Red-O-stained liver sections from: $(A)$ basal diet; $(B)$ basal diet with betaine administration; (C) high-fat diet; (D) high-fat diet with betaine administration. Values are means $(n 5)$, with standard deviations. ${ }^{\mathrm{a}, \mathrm{b}, \mathrm{c}}$ Mean values with unlike superscript letters were significantly different $(P<0.05)$. Groups: T1, basal diet; T2, basal diet with betaine administration; T3, highfat diet; T4, high-fat diet with betaine administration. A colour version of this figure can be found online at http://www.journals.cambridge.org/bjn

Betaine increased the activity, gene and protein expression of fibroblast growth factor 21, and elevated the gene expression of AMP-activated protein kinase in the liver

The changes of betaine administration on the activity, gene and protein expression of FGF21 in the liver are shown in Fig. 5(A)-(C). Compared with rats fed the basal diet (T1), the high-fat diet (T3) elevated $(P<0.05)$ the gene expression of hepatic FGF21. Betaine administration significantly increased $(P<0.05)$ the concentration and gene expression of hepatic FGF21 in both the basal diet (T1) and high-fat diet feeding (T3) groups. Additionally, compared with rats fed with a high-fat diet (T3), oral administration of betaine in the high-fat group (T4) significantly increased $(P<0.05)$ the protein expression of FGF21. Regarding AMP-activated protein kinase (AMPK), compared with rats fed with the basal diet (T1), the high-fat (T3) diet increased $(P<0.05)$ the mRNA expression of $A M P K$ (Fig. 5(D)). Oral administration of betaine in the high-fat diet group (T4) significantly increased $(P<0.05)$ the gene expression of $A M P K$ compared with high-fat diet-fed rats (T3). The gene expression of $A M P K$ in the basal diet-treated group (T1) was not affected by betaine administration.

\section{Discussion}

The present study indicated that betaine did not affect body weight in rats fed with either basal diet or high-fat diet. Variable effects of betaine on body weight were previously reported $^{(21,27,28)}$. These differences might be attributed to the diversity of animals, dietary nutrients, and trial periods, etc. TAG, cholesterol, lecithin and NEFA were the major components of plasma lipids. In this investigation, treatments of high-fat diet significantly increased the serum concentrations of TAG, lecithin and glucose, which suggested that high-fat diet might result in hyperlipidaemia. It is well-established that the intake of SFA is associated with hyperlipidaemia, diabetes and $\mathrm{CVD}^{(29)}$. In the present study, compared with basal diet, the high-fat diet supplemented with lard oil increased the energy content as well as the SFA content in high-fat diet. Therefore, the increase in serum lipid maybe associated with the difference of fatty acid composition in diets. The hyperlipidaemia caused by high-fat diet was not relieved by betaine administration in our experiment, which increased the levels of serum TAG, lecithin and glucose further compared with those of high-fat diet-fed rats. This is in line with a previous research ${ }^{(30)}$, which suggested that betaine-supplemented pigs exhibited significantly higher plasma concentrations of TAG, phospholipids and lipoprotein cholesterol. Therefore, the effect of betaine in these parameters suggested that betaine might enhance lipids transportation accompanied with dyslipidaemia risk $^{(31)}$. The glucose increase induced by betaine was observed in basal diet-fed rats and high-fat dietfed rats. Accordingly, it is suspected that the increase in serum glucose was due to betaine, which improved lipid and energy metabolism accompanied with decreasing uptake of serum glucose by peripheral tissues; this needs to

Table 5. Effects of betaine on hepatic lipid metabolism (Mean values and standard deviations, $n 7$ )

\begin{tabular}{|c|c|c|c|c|c|c|c|c|}
\hline & \multicolumn{2}{|c|}{$\mathrm{T} 1$} & \multicolumn{2}{|c|}{$\mathrm{T} 2$} & \multicolumn{2}{|c|}{ T3 } & \multicolumn{2}{|c|}{$\mathrm{T} 4$} \\
\hline & Mean & SD & Mean & SD & Mean & SD & Mean & SD \\
\hline TAG (mg/g) & $7 \cdot 81^{\mathrm{b}}$ & 0.66 & $7.47^{\mathrm{b}}$ & 0.58 & $9 \cdot 20^{a}$ & 1.42 & $7.96^{\mathrm{b}}$ & 0.84 \\
\hline NEFA ( $\mu \mathrm{mol} / \mathrm{g}$ protein) & $32 \cdot 73^{\mathrm{b}}$ & $9 \cdot 16$ & $39 \cdot 44^{\mathrm{b}}$ & 11.77 & $57.93^{\mathrm{a}}$ & $12 \cdot 76$ & $67 \cdot 08^{a}$ & $12 \cdot 27$ \\
\hline $\mathrm{TC}(\mathrm{mg} / \mathrm{g})$ & $2 \cdot 21^{\mathrm{b}, \mathrm{c}}$ & $0 \cdot 17$ & $2 \cdot 46^{\mathrm{a}, \mathrm{c}}$ & 0.47 & $2 \cdot 58^{\mathrm{a}, \mathrm{c}}$ & 0.48 & $2 \cdot 76^{a}$ & 0.49 \\
\hline Lecithin (ng/g) & $1.00^{\mathrm{C}}$ & 0.05 & $1 \cdot 10^{b}$ & 0.05 & $1.04^{\mathrm{c}}$ & 0.05 & $1.17^{\mathrm{a}}$ & 0.02 \\
\hline VLDL $(\mathrm{mg} / \mathrm{g})$ & $1 \cdot 13$ & 0.05 & $1 \cdot 18$ & 0.05 & $1 \cdot 13$ & 0.06 & 1.16 & 0.07 \\
\hline Carnitine $(\mu \mathrm{mol} / \mathrm{g})$ & $2 \cdot 63$ & 0.25 & $2 \cdot 78$ & 0.14 & $2 \cdot 56$ & 0.35 & $2 \cdot 60$ & 0.22 \\
\hline
\end{tabular}

T1, basal diet; T2, basal diet with betaine administration; T3, high-fat diet; T4, high-fat diet with betaine administration; TC, total cholesterol.

${ }^{\mathrm{a}, \mathrm{b}, \mathrm{c}}$ Mean values with unlike superscript letters were significantly different $(P<0.05)$ 

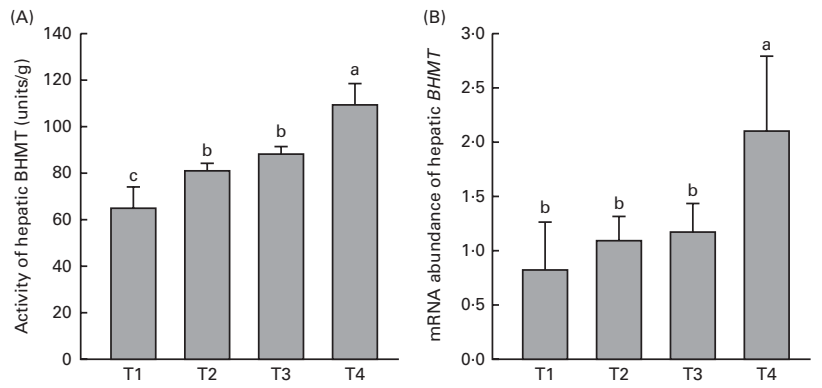

Fig. 3. (A) Effect of betaine on the activity of betaine-homocysteine methyltransferase (BHMT) in the liver. (B) Effect of betaine on the mRNA abundance of $B H M T$ in the liver. Values are means $(n 7)$, with standard deviations represented by vertical bars. ${ }^{\mathrm{a}, \mathrm{b}, \mathrm{c}}$ Mean values with unlike letters were significantly different $(P<0.05)$. Groups: $\mathrm{T} 1$, basal diet; $\mathrm{T} 2$, basal diet with betaine administration; T3, high-fat diet; T4, high-fat diet with betaine administration.

be confirmed by further research. Our data also showed that treatment of high-fat diet significantly increased hepatic vesicular fat accumulation and Oil-Red-O-stained area, respectively. Moreover, the concentrations of TAG and NEFA in the liver were significantly increased in the group that was given the high-fat diet, indicating that high-fat diet treatment for 4 weeks led to hepatic lipid accumulation in rats. In the present study, oral administration of betaine reduced the Oil-Red-O-stained area, and normalised the concentration of hepatic TAG in rats fed with the high-fat diet, suggesting that hepatic lipid accumulation induced by high-fat diet was reversed by betaine administration.

The present study also investigated the effect of betaine on the factors playing a vital role in protecting the liver against lipid accumulation, which were altered by betaine administration. BHMT plays a critical role in the hepatic one-carbon metabolism, and converts homocysteine to methionine using betaine as a methyl donor ${ }^{(32)}$. Significant increase in BHMT activity was observed in betaine administration rats given either basal diet or high-fat diet, which was accompanied with the notable increase of BHMT mRNA abundance in the high-fat group. Similarly, Kharbanda et al. ${ }^{(33)}$ reported a significant increase in enzyme activity and mRNA abundance of BHMT in rats fed diets supplemented with $1 \%$ betaine for 4 weeks. Feng et al. $^{(34)}$ also reported that BHMT activity was significantly increased when pigs were offered $0 \cdot 125 \%$ betaine. It has been well-documented that betaine supplementation enhances recycling of homocysteine for the generation of methionine and $S$-adenosylmethionine, and the increase of hepatic $S$-adenosylmethionine results in attenuation of fatty liver, by activating a cascade of events including phosphatidylcholine synthesis, formation of VLDL and transportation of hepatic lipid ${ }^{(33,35)}$. Our results showed that betaine administration elevated the levels of hepatic lecithin and serum lecithin and VLDL in high-fat diet-fed rats, which suggested that betaine reversed hepatic lipid accumulation by elevated lecithin and VLDL levels via increasing the expression and activity of BHMT, thus enhancing hepatic lipid export. Besides, high-fat diet also increased the activity of BHMT in the liver. This is in agreement with Deminice et al. ${ }^{(36)}$, who reported that high-fat diet treatment for 3 weeks elevated the mRNA level of BHMT. Therefore, high-fat diet might induce the enhancement of hepatic lipid export.

Owing to the specific property, CPT1 is a key rate-limiting enzyme of $\beta$-oxidation ${ }^{(37)}$, and is reported to be a target of $\operatorname{PPAR} \alpha^{(38)}$. PPAR $\alpha$, a nuclear receptor, is activated by fatty acids, and it regulates the transcription of numerous genes encoding enzymes in fatty acid oxidation and transportation $^{(39,40)}$. Enzyme analysis showed that betaine administration had no apparent effect on the activity of CPT1 in rats fed with basal diet. This is in agreement with Huang et $a l .{ }^{(41)}$, who reported that an effect of betaine on the activity of CPT1 in liver was not observed. In the present study, downregulations of PPAR $\alpha$ and its target (CPT1) were observed in the treatments of high-fat diet, which is consistent with the study of Deminice et $a l{ }^{(36)}$, who reported that high-fat diet reduced mRNA levels for PPAR $\alpha$ as well as its downstream targets CPT1. Oral administration of betaine in the high-fat diet rats obviously elevated the gene expression of PPAR $\alpha$ and normalised the gene expression of CPT1 in the present study. Wang et al. ${ }^{(40)}$ reported a similar result, which demonstrated that betaine supplementation ( $2 \%$ betaine/100 g diet) increased both PPAR $\alpha$ and CPT1 expressions of apoE ${ }^{-/-}$ mice. The gene expression of CPT1 in liver was not altered by betaine supplement, as reported by Huang et al. ${ }^{(41)}$, but, interestingly, betaine reversed the inhibition of CPT1 induced by high-fat diet in the present study. Therefore, betaine may induce the alteration of CPT1 gene expression in specific conditions, as in apoE $\mathrm{E}^{-/-}$mice and high-fat feeding rats as mentioned above, which needs the support of further
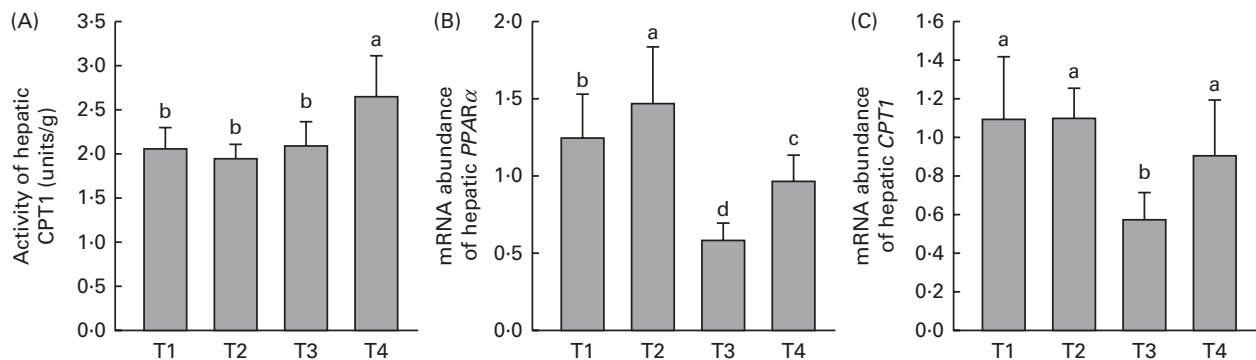

Fig. 4. (A) Effect of betaine on the activity of carnitine palmitoyltransferase 1 (CPT1) in the liver. (B) Effect of betaine on the gene expression of PPAR $\alpha$ in the liver. (C) Effect of betaine on the gene expression of $C P T 1$ in the liver. Values are means $(n 7)$, with standard deviations represented by vertical bars. ${ }^{\text {ab, }, \mathrm{c}, \mathrm{d}}$ Mean values with unlike letters were significantly different $(P<0.05)$. Groups: T1, basal diet; T2, basal diet with betaine administration; T3, high-fat diet; T4, high-fat diet with betaine administration. 

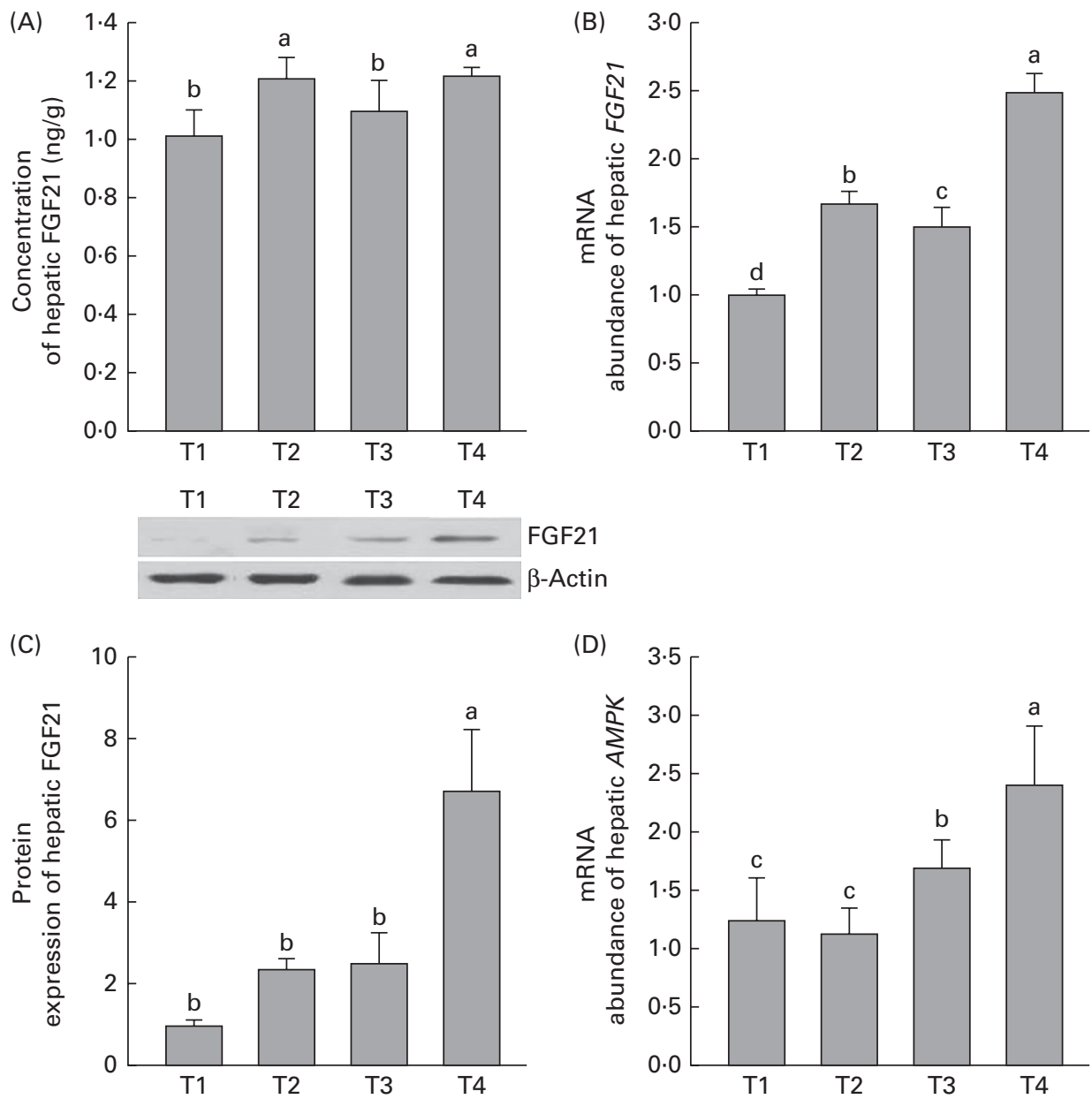

Fig. 5. (A) Effects of betaine on the content of fibroblast growth factor 21 (FGF21) in the liver. (B) Effects of betaine on the FGF21 mRNA abundance in the liver. (C) Effects of betaine on relative protein expression of FGF21 in the liver. (D) Effects of betaine on the mRNA abundance of AMP-activated protein kinase $(A M P K)$. Band intensities were determined by using AlphaEase FC analysis software (Alpha Innotech). Values are means $(n 7)$, with standard deviations represented by vertical bars. ${ }^{\text {a,b,c,d }}$ Mean values with unlike letters were significantly different $(P<0.05)$. Groups: T1, basal diet; T2, basal diet with betaine administration; T3, high-fat diet; T4, high-fat diet with betaine administration.

research. Moreover, it is well-established that AMPK indirectly increased CPT1 activity by inhibiting acetyl-CoA carboxylase activity $^{(42,43)}$. Our data indicated that betaine significantly elevated hepatic $A M P K$ gene expression (Fig. 5(D)), and there was a consistent response to betaine between gene expressions of $A M P K$ and $C P T 1$. In the present study, betaine reversed the inhibition of $C P T 1$ gene expression induced by high-fat diet treatment, which may be due to the activation of AMPK. It can be presumed that betaine normalised fatty acid oxidation in rats fed with a high-fat diet via activating AMPK and up-regulating PPAR $\alpha$ and CPT1 gene expression, thus preventing lipid accumulation in the liver.

FGF21, a novel metabolic regulator, is produced predominantly in the liver, and involved in the regulation of lipid metabolism, including lipolysis, fatty acid oxidation and ketogenesis $^{(44-48)}$. In the present study, FGF21 concentration as well as its gene and protein expressions were significantly increased in betaine administration groups. FGF21 can be tightly regulated by nutrition in animal models, and previous research indicated that 2 weeks of $5 \%$ betaine feeding increased FGF21 expression 7 -fold in the liver of mice ${ }^{(49)}$, which suggested that betaine elevated indeed the expression of FGF21. However, the relationship between BHMT and FGF21 is not clear, since the results of the present study showed that betaine administration resulted in elevation of BHMT and FGF21, whereas a previous study indicated that $\mathrm{BHMT}^{-/-}$mice increased FGF21 expression $^{(46)}$. Therefore, it can be speculated that $\mathrm{BHMT}^{-/-}$ mice may increase FGF21 expression via the elevation of betaine concentration instead of the direct regulation by BHMT. Chau et al. ${ }^{(50)}$ demonstrated that FGF21 regulates energy homeostasis through an AMPK-SIRT1 (sirtuin 1)-PGC1 $\alpha$ (peroxisome proliferator-activated receptor- $\gamma$ co-activator $1 \alpha$ )-dependent mechanism in adipocytes. In the present study, expressions of FGF21 and AMPK were observed to be positively interrelated in response to betaine treatment. AMPK is a heterotrimeric protein that serves as a sensor of cellular energy levels, and it plays a critical role in the regulation of lipid metabolism by stimulating fatty acids oxidation, and inhibiting their synthesis ${ }^{51,52)}$. Therefore, it can be speculated that betaine may activate AMPK system through up-regulating FGF21 expression, thus enhancing lipolysis in the liver. Additionally, FGF21 was reported to be regulated by PPAR $\alpha^{(44)}$, expressions 
of FGF21 and PPAR $\alpha$ were observed to be positively interrelated in response to betaine treatment as well. Therefore, betaine might increase the expression of FGF21 via up-regulating PPAR $\alpha$ in the high-fat group.

In summary, the present study indicated that betaine could alleviate hepatic lipid accumulation induced by a high-fat diet via enhancing hepatic lipid export and fatty acid oxidation. In high-fat diet-fed rats, betaine could elevate the lecithin and VLDL levels via enhancing the expression of BHMT, thus improving hepatic lipid export; meanwhile, betaine may increase the expression of CPT1 via activating PPAR $\alpha$, FGF21 and AMPK, thus enhancing fatty acids oxidation. Betaine could induce changes in the key factors involved in alleviating hepatic lipid accumulation, and the mechanism by which betaine regulates these key factors needs to be confirmed by further research studies.

\section{Acknowledgements}

This project was funded and supported by the National Basic Research Program of China (2012CB124705). The funding agency had no role in the design, analysis or writing of this article. The present research received no specific grant from any funding agency of commercial or not-for-profit sectors.

The authors' contributions are as follows: J. F. and Y. W. were in charge of the whole trial; L. X., D. H., Q. H., J. W. and J. F. conducted the research; L. X., D. H., and J. W. analysed the data and wrote the manuscript.

The authors have no conflicts of interest to declare.

\section{References}

1. Cholewa JM, Guimarães-Ferreira L \& Zanchi NE (2014) Effects of betaine on performance and body composition: a review of recent findings and potential mechanisms. Amino Acids 46, 1785-1793.

2. Craig SA (2004) Betaine in human nutrition. Am J Clin Nutr 80, 539-549.

3. Kim SK \& Kim YC (2005) Effects of betaine supplementation on hepatic metabolism of sulfur-containing amino acids in mice. J Hepatol 42, 907-913.

4. Ratriyanto A, Mosenthin R, Bauer E, et al. (2009) Metabolic, osmoregulatory and nutritional functions of betaine in monogastric animals. Asian-Austral J Animal Sci 22 $1461-1476$.

5. Eklund M, Bauer E, Wamatu J, et al. (2005) Potential nutritional and physiological functions of betaine in livestock. Nutr Res Rev 18, 31-48.

6. Lever M \& Slow S (2010) The clinical significance of betaine, an osmolyte with a key role in methyl group metabolism. Clin Biochem 43, 732-744.

7. Ganesan B, Buddhan S, Anandan R, et al. (2010) Antioxidant defense of betaine against isoprenaline-induced myocardial infarction in rats. Mol Biol Rep 37, 1319-1327.

8. van Wettere W, Herde P \& Hughes P (2012) Supplementing sow gestation diets with betaine during summer increases litter size of sows with greater numbers of parities. Anim Reprod Sci 132, 44-49.

9. Sales J (2011) A meta-analysis of the effects of dietary betaine supplementation on finishing performance and carcass characteristics of pigs. Anim Feed Sci Tech 165, 68-78.
10. Huang Q-C, Xu Z-R, Han X-Y, et al. (2006) Changes in hormones, growth factor and lipid metabolism in finishing pigs fed betaine. Livest Sci 105, 78-85.

11. Wang Y, Xu Z \& Feng J (2004) The effect of betaine and DL-methionine on growth performance and carcass characteristics in meat ducks. Anim Feed Sci Tech 116, 151-159.

12. Abdelmalek MF, Angulo P, Jorgensen RA, et al. (2001) Betaine, a promising new agent for patients with nonalcoholic steatohepatitis: results of a pilot study. $A m J$ Gastroenterol 96, 2711-2717.

13. Abdelmalek MF, Sanderson SO, Angulo P, et al. (2010) Correction: Betaine for nonalcoholic fatty liver disease: results of a randomized placebo-controlled trial. Hepatology 51, 1818-1826.

14. Lelliott C \& Vidal-Puig A (2004) Lipotoxicity, an imbalance between lipogenesis de novo and fatty acid oxidation. Int J Obes Relat Metab Disord 28, S22-S28.

15. Browning JD \& Horton JD (2004) Molecular mediators of hepatic steatosis and liver injury. J Clin Invest 114, 147-152.

16. Fabbrini E, Sullivan S \& Klein S (2010) Obesity and nonalcoholic fatty liver disease: biochemical, metabolic, and clinical implications. Hepatology 51, 679-689.

17. Yang H, Li F, Kong X, et al. (2012) Molecular cloning, tissue distribution and ontogenetic expression of Xiang pig Chemerin and its involvement in regulating energy metabolism through Akt and ERK1/2 signaling pathways. Mol Biol Rep 39, 1887-1894.

18. Zhang W, Wang L-W, Wang L-K, et al. (2013) Betaine protects against high-fat-diet-induced liver injury by inhibition of high-mobility group box 1 and Toll-like receptor 4 expression in rats. Dig Dis Sci 58, 3198-3206.

19. Jung YS, Kim SJ, Kwon DY, et al. (2013) Alleviation of alcoholic liver injury by betaine involves an enhancement of antioxidant defense via regulation of sulfur amino acid metabolism. Food Chem Toxicol 62, 292-298.

20. Song Z, Deaciuc I, Zhou Z, et al. (2007) Involvement of AMP-activated protein kinase in beneficial effects of betaine on high-sucrose diet-induced hepatic steatosis. Am J Physiol Gastrointest Liver Physiol 293, G894-G902.

21. Kwon DY, Jung YS, Kim SJ, et al. (2009) Impaired sulfuramino acid metabolism and oxidative stress in nonalcoholic fatty liver are alleviated by betaine supplementation in rats. J Nutr 139, 63-68.

22. Kim SJ, Jung YS, Kwon DY, et al. (2008) Alleviation of acute ethanol-induced liver injury and impaired metabolomics of $S$-containing substances by betaine supplementation. Biochem Biophys Res Commun 368, 893-898.

23. Alirezaei M, Jelodar G, Ghayemi Z, et al. (2014) Antioxidant and methyl donor effects of betaine versus ethanol-induced oxidative stress in the rat liver. Comp Clin Pathol 23, $161-168$.

24. Reeves PG, Nielsen FH \& Fahey GC Jr (1993) AIN-93 purified diets for laboratory rodents: final report of the American Institute of Nutrition ad hoc writing committee on the reformulation of the AIN-76A rodent diet. J Nutr 123, 1939-1951.

25. Livak KJ \& Schmittgen TD (2001) Analysis of relative gene expression data using real-time quantitative PCR and the $2^{-\Delta \Delta \mathrm{CT}}$ method. Methods 25, 402-408.

26. Duan Y, Li F, Li L, et al. (2014) n-6:n-3 PUFA ratio is involved in regulating lipid metabolism and inflammation in pigs. $\mathrm{BrJ}$ Nutr 111, 445-451.

27. Wang Z, Yao T, Pini M, et al. (2010) Betaine improved adipose tissue function in mice fed a high-fat diet: a mechanism for hepatoprotective effect of betaine in nonalcoholic fatty liver disease. Am J Physiol Gastrointest Liver Physiol 298, G634-G642. 
28. Wray-Cahen D, Fernandez-Figares I, Virtanen E, et al. (2004) Betaine improves growth, but does not induce whole body or hepatic palmitate oxidation in swine (Sus scrofa domestica). Comp Biochem Physiol A Mol Integr Physiol 137, 131-140.

29. Ros E \& Mataix J (2006) Fatty acid composition of nuts implications for cardiovascular health. Br J Nutr 96, S29-S35.

30. Martins JM, Neves JA, Freitas A, et al. (2010) Betaine supplementation affects the cholesterol but not the lipid profile of pigs. Eur J Lipid Sci Tech 112, 295-303.

31. Olthof M \& Verhoef P (2005) Effects of betaine intake on plasma homocysteine concentrations and consequences for health. Curr Drug Metab 6, 15-22.

32. Teng Y-W, Mehedint MG, Garrow TA, et al. (2011) Deletion of betaine-homocysteine $S$-methyltransferase in mice perturbs choline and 1-carbon metabolism, resulting in fatty liver and hepatocellular carcinomas. $J$ Biol Chem 286, 36258-36267.

33. Kharbanda KK, Mailliard ME, Baldwin CR, et al. (2007) Betaine attenuates alcoholic steatosis by restoring phosphatidylcholine generation via the phosphatidylethanolamine methyltransferase pathway. J Hepatol 46, 314-321.

34. Feng J, Liu X, Wang Y, et al. (2006) Effects of betaine on performance, carcass characteristics and hepatic betainehomocysteine methyltransferase activity in finishing barrows. Asian-Austral J Animal Sci 19, 402.

35. Zhu X, Song J, Mar M, et al. (2003) Phosphatidylethanolamine $N$-methyltransferase (PEMT) knockout mice have hepatic steatosis and abnormal hepatic choline metabolite concentrations despite ingesting a recommended dietary intake of choline. Biochem J 370, 987-993.

36. Deminice R, da Silva RP, Lamarre SG, et al. (2011) Creatine supplementation prevents the accumulation of fat in the livers of rats fed a high-fat diet. J Nutr 141, 1799-1804.

37. Korman SH, Waterham HR, Gutman A, et al. (2005) Novel metabolic and molecular findings in hepatic carnitine palmitoyltransferase I deficiency. Mol Genet Metab 86, 337-343.

38. Ferré P (2004) The biology of peroxisome proliferator-activated receptors: relationship with lipid metabolism and insulin sensitivity. Diabetes 53, S43-S50.

39. Yang H, Li F, Xiong X, et al. (2013) Soy isoflavones modulate adipokines and myokines to regulate lipid metabolism in adipose tissue, skeletal muscle and liver of male Huanjiang mini-pigs. Mol Cell Endocrinol 365, 44-51.

40. Wang L, Chen L, Tan Y, et al. (2013) Betaine supplement alleviates hepatic triglyceride accumulation of apolipoprotein E deficient mice via reducing methylation of peroxisomal proliferator-activated receptor alpha promoter. Lipids Health Dis 12, 34

41. Huang Q-C, Han X-Y, Xu Z-R, et al. (2009) Betaine suppresses carnitine palmitoyltransferase I in skeletal muscle but not in liver of finishing pigs. Livest Sci 126, 130-135.

42. Assifi MM, Suchankova G, Constant S, et al. (2005) AMPactivated protein kinase and coordination of hepatic fatty acid metabolism of starved/carbohydrate-refed rats. $A m J$ Physiol Endocrinol Metab 289, E794-E800.

43. Xue B \& Kahn BB (2006) AMPK integrates nutrient and hormonal signals to regulate food intake and energy balance through effects in the hypothalamus and peripheral tissues. J Physiol 574, 73-83.

44. Badman MK, Pissios P, Kennedy AR, et al. (2007) Hepatic fibroblast growth factor 21 is regulated by PPAR $\alpha$ and is a key mediator of hepatic lipid metabolism in ketotic states. Cell Metab 5, 426-437.

45. Kharitonenkov A, Shiyanova TL, Koester A, et al. (2005) FGF-21 as a novel metabolic regulator. I Clin Invest $\mathbf{1 1 5}$, $1627-1635$.

46. Uebanso T, Taketani Y, Fukaya M, et al. (2009) Hypocaloric high-protein diet improves fatty liver and hypertriglyceridemia in sucrose-fed obese rats via two pathways. Am J Physiol Endocrinol Metab 297, E76-E84.

47. Xu J, Lloyd DJ, Hale C, et al. (2009) Fibroblast growth factor 21 reverses hepatic steatosis, increases energy expenditure, and improves insulin sensitivity in diet-induced obese mice. Diabetes 58, 250-259.

48. Coskun T, Bina HA, Schneider MA, et al. (2008) Fibroblast growth factor 21 corrects obesity in mice. Endocrinology 149, 6018-6027.

49. Teng Y-W, Ellis JM, Coleman RA, et al. (2012) Mouse betainehomocysteine $S$-methyltransferase deficiency reduces body fat via increasing energy expenditure and impairing lipid synthesis and enhancing glucose oxidation in white adipose tissue. J Biol Chem 287, 16187-16198.

50. Chau MD, Gao J, Yang Q, et al. (2010) Fibroblast growth factor 21 regulates energy metabolism by activating the AMPK-SIRT1-PGC-1 $\alpha$ pathway. Proc Natl Acad Sci U S A 107, 12553-12558.

51. Lee WJ, Kim M, Park H-S, et al. (2006) AMPK activation increases fatty acid oxidation in skeletal muscle by activating PPAR $\alpha$ and PGC-1. Biochem Biophys Res Commun 340, 291-295

52. Li Y, Xu S, Mihaylova MM, et al. (2011) AMPK phosphorylates and inhibits SREBP activity to attenuate hepatic steatosis and atherosclerosis in diet-induced insulin-resistant mice. Cell Metab 13, 376-388. 